\title{
Evidence for Secondary Flux Rope Generated by the Electron Kelvin-Helmholtz Instability in a Magnetic Reconnection Diffusion Region
}

\author{
Z. H. Zhong, ${ }^{1,2,3}$ R. X. Tang, ${ }^{1,2, *}$ M. Zhou, ${ }^{2,4, \dagger}$ X. H. Deng, ${ }^{2, \ddagger}$ Y. Pang, ${ }^{2}$ W. R. Paterson, ${ }^{5}$ B. L. Giles, ${ }^{5}$ \\ J. L. Burch, ${ }^{6}$ R. B. Tobert, ${ }^{7}$ R. E. Ergun, ${ }^{8}$ Y. V. Khotyaintsev, ${ }^{9}$ and P.-A. Lindquist ${ }^{10}$ \\ ${ }^{1}$ Department of Physics, School of Science, Nanchang University, Nanchang 330031, People's Republic of China \\ ${ }^{2}$ Institute of Space Science and Technology, Nanchang University, Nanchang 330031, People's Republic of China \\ ${ }^{3}$ School of Resources Environmental and Chemical Engineering, Nanchang University, Nanchang 330031, People's Republic of China \\ ${ }^{4}$ Department of Physics and Astronomy, University of California, Los Angeles, California 90095, USA \\ ${ }^{5}$ NASA, Goddard Space Flight Center, Greenbelt, Maryland 20771, USA \\ ${ }^{6}$ Southwest Research Institute, San Antonio Texas 78238, USA \\ ${ }^{7}$ University of New Hampshire, Durham, New Hampshire 03824, USA \\ ${ }^{8}$ University of Colorado LASP, Boulder, Colorado 80303, USA \\ ${ }^{9}$ Swedish Institute of Space Physics, Uppsala 75121, Sweden \\ ${ }^{10}$ Royal Institute of Technology, Stockholm SE-11428, Sweden
}

(Received 18 August 2017; published 13 February 2018)

\begin{abstract}
Secondary flux ropes are suggested to play important roles in energy dissipation and particle acceleration during magnetic reconnection. However, their generation mechanism is not fully understood. In this Letter, we present the first direct evidence that a secondary flux rope was generated due to the evolution of an electron vortex, which was driven by the electron Kelvin-Helmholtz instability in an ion diffusion region as observed by the Magnetospheric Multiscale mission. The subion scale (less than the ion inertial length) flux rope was embedded within the electron vortex, which contained a secondary electron diffusion region at the trailing edge of the flux rope. We propose that intense electron shear flow produced by reconnection generated the electron Kelvin-Helmholtz vortex, which induced a secondary reconnection in the exhaust of the primary $X$ line and then led to the formation of the flux rope. This result strongly suggests that secondary electron Kelvin-Helmholtz instability is important for reconnection dynamics.
\end{abstract}

DOI: $10.1103 /$ PhysRevLett.120.075101

Magnetic reconnection is a fundamental physical process in many different plasma systems. It efficiently converts magnetic energy into kinetic and thermal energy of plasma. One outstanding question of reconnection is where and how the magnetic energy is dissipated. It has been shown that flux ropes (FRs) likely play essential roles in the energy dissipation process during reconnection by energizing particles, regulating the reconnection rate, and providing significant energy dissipation as they are coalescing [1-5]. Secondary FRs, which are ion-scale FRs, have received much attention due to their close relation to the electron physics in reconnection [6-8].

The generation of secondary FRs is not fully understood yet. One well-known mechanism for generating secondary FR is the tearing instability in extended electron-scale current layers [6,7]. A secondary FR observed in an ion diffusion region in Earth's magnetotail supports this

Published by the American Physical Society under the terms of the Creative Commons Attribution 4.0 International license. Further distribution of this work must maintain attribution to the author(s) and the published article's title, journal citation, and DOI. scenario [9]. On the other hand, kinetic simulations show that secondary FR can be generated by electron vortex, which is a nonlinear consequence of electron KelvinHelmholtz (K-H) instability driven by electron shear flows produced during magnetic reconnection [10,11]. However, this scenario has not been confirmed yet.

NASA's Magnetospheric Multiscale (MMS) mission [12] consists of four identical spacecraft with interspacecraft distance varying between the ion and electron scale. With high-resolution particle distribution and electromagnetic field measurements, it provides unprecedented opportunity to uncover the electron physics associated with reconnection. In this Letter, we report the first direct evidence that secondary FR can be generated as a result of the evolution of the electron $\mathrm{K}-\mathrm{H}$ vortex in the reconnection ion diffusion region (IDR). One subion scale (less than the ion inertial length) FR and an electron diffusion region (EDR) were observed within an electron vortex during an asymmetric reconnection at the dayside magnetopause (MP). Data used in this study are from the following instruments of the MMS: the Fluxgate Magnetometer (FGM) [13], the Fast Plasma instrument (FPI) [14], and the Electric Double Probe (EDP) [15,16]. 


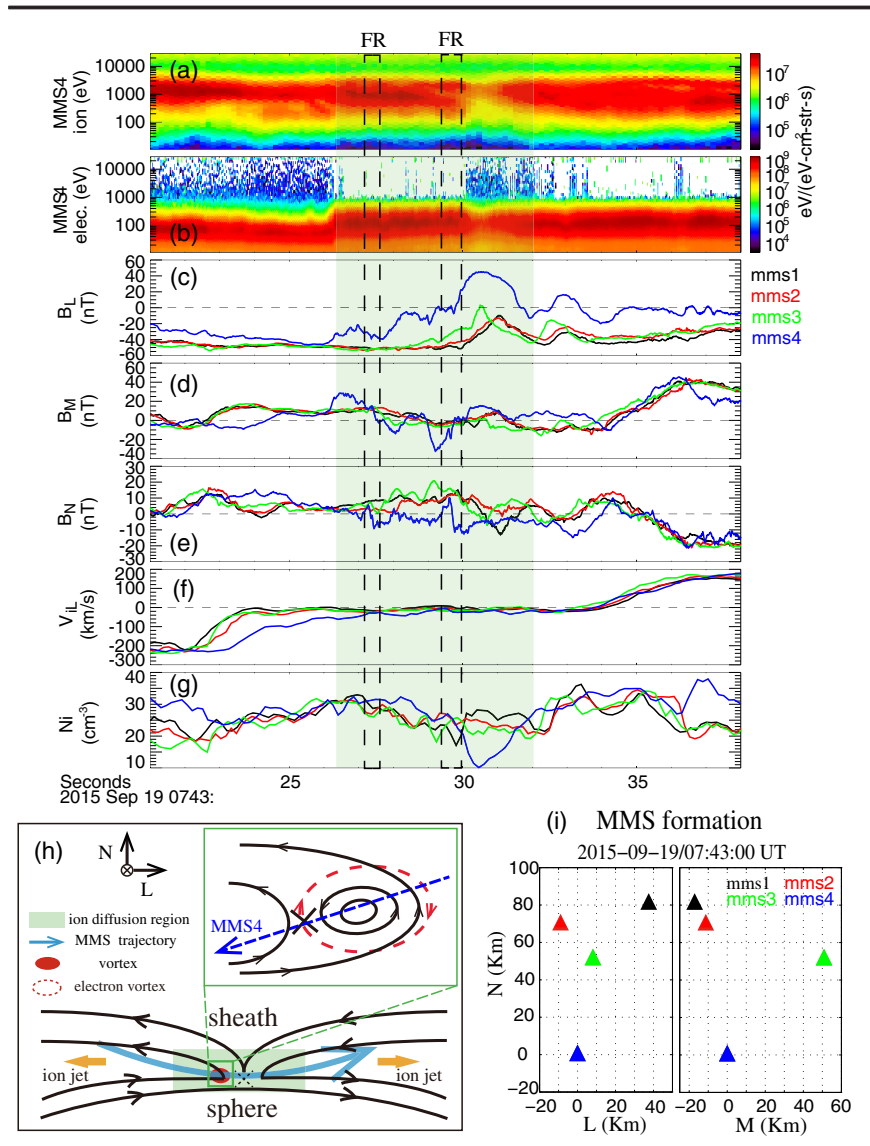

FIG. 1. Overview of MMS observations of the diffusion region between 07:43:21 and 07:43:38 UT on September 19, 2015. (a) and (b) Ion and electron differential energy fluxes observed by MMS4, (c)-(e) $L, M$, and $N$ components of the magnetic filed observed by four MMS spacecraft, (f) $L$ component of the ion bulk velocity, (g) ion density, and (h) a schematic of the inferred trajectory of MMS through the reconnection site. Top right inset in (h) zooms in on the FR and vortex, which were moving in the $+\mathbf{L}$ direction. The shadow in panels (a) $-(\mathrm{g})$ marks the IDR. Panel (i) shows the relative positions of the MMS spacecraft at 07:43:00 UT in the LMN coordinates.

Figure 1 presents the overview of all four MMS spacecraft measurements during 07:43:21-07:43:38 UT when they were around $[6.3,5.4,-3.0] R_{E}$ in Geocentric Solar Magnetospheric (GSM) coordinates. We transferred the vectors into the local boundary normal coordinates, which are obtained by the minimum variance analysis (MVA) [17] on magnetic fields during one magnetopause (MP) crossing between 07:44 and 07:45 UT observed by MMS4. $N$ points outward along the MP normal, $\boldsymbol{L}$ is the maximum variation direction that points along the reconnecting magnetic field component, and $\boldsymbol{M}$ completes a right-handed orthogonal coordinate system. The transformation from GSM to LMN coordinates is given by $\boldsymbol{L}=[0.065,0.752,0.656], \quad \boldsymbol{M}=[0.790,-0.441,0.427]$, and $\boldsymbol{N}=[0.610,0.490,-0.622]$. The four MMS spacecraft formed a tetrahedron in space with separation about 1 to 2 $d_{i} \quad\left(1 \quad d_{i} \sim 46 \mathrm{~km}\right.$ given the average plasma density $\sim 25 \mathrm{~cm}^{-3}$ between 07:43:22 and 07:43:30 UT). The relative positions of MMS are shown in Fig. 1(i).

During this interval, MMS observed a flow reversal in $V_{i L}$ from -250 to $160 \mathrm{~km} / \mathrm{s}$. This indicates that a reconnection $X$ line passed the MMS along the $-\mathbf{L}$ direction. Magnetic field $B_{L}$ component of MMS4 changed from negative to positive, and then returned back to negative. This indicates that MMS4 crossed the MP from the magnetosheath $\left(B_{L}<0\right)$ to the magnetosphere $\left(B_{L}>0\right)$, and moved back to the magnetosheath. The MP crossing is also evident in the plasma density variation as $N_{i}$ decreases towards the magnetospheric side. MMS4 did not enter into the magnetosphere proper but mostly in the exhaust region since the plasma density on the magnetospheric side is higher than the value in the magnetosphere proper, and the ion and electron energy spectrograms show mixed populations from both sides of the MP. The other three MMS spacecraft were in the $+\mathbf{N}$ direction with respect to MMS4 and did not cross the MP current sheet where $B_{L}=0$.

All four spacecraft detected an IDR from 07:43:26 to 07:43:32 UT. The IDR was identified by the signature of unmagnetized ions which were accelerated by the reconnection electric field $[18,19]$. The small value of $V_{i L}$ also supports that MMS was near the $X$ line since ion flow speed in the IDR is much smaller than that in the exhaust further away from the $X$ line. Furthermore, an EDR associated with the reconnection $X$ line was encountered by MMS3 at 07:43:30.3 UT. This EDR has been identified and discussed in detail in Ref. [18]. MMS4 detected two small-scale FRs (marked by the dashed boxes in Fig. 1) within the IDR; hence, they were secondary FRs according to the terminology in previous literature [6,8-9]. One FR was detected in the magnetosheath side of the MP and the other one was detected in the MP current sheet. Both FRs were manifested by the bipolar variations in $B_{N}$ [Fig. 1(e)] and enhancements in the out-of-plane component $B_{M}$ [Fig. 1(d)]. However, both FRs were not encountered by the other three spacecraft. Figure 1(h) presents a schematic of the inferred trajectory of the MMS spacecraft through this reconnection site.

We focus on the second FR since it was observed in the MP current sheet and close to the EDR observed by MMS3. Figure 2 presents the magnetic fields and electron bulk flows associated with this FR. The bipolar $B_{N}$ variation is from positive to negative, which suggests that this FR moved in the $+\mathbf{L}$ direction. This is not a force-free FR since the perpendicular current is comparable to the parallel current within the FR [see Fig. 2(h)]. One intriguing feature of this FR is that it was associated with significant electron flow variation. $V_{e L}$ [Fig. 2(e)] and $V_{e N}$ [Fig. 2(g)] reverses from 500 to $-500 \mathrm{~km} / \mathrm{s}$ and -600 to $450 \mathrm{~km} / \mathrm{s}$, respectively, while $V_{e M}$ [Fig. 2(f)] exhibits two positive peaks: $600 \mathrm{~km} / \mathrm{s}$ at 07:43:29.3 UT and $750 \mathrm{~km} / \mathrm{s}$ at 07:43:29.9 UT. Similar to the FR, these large amplitude flows were not detected by the other three spacecraft. 


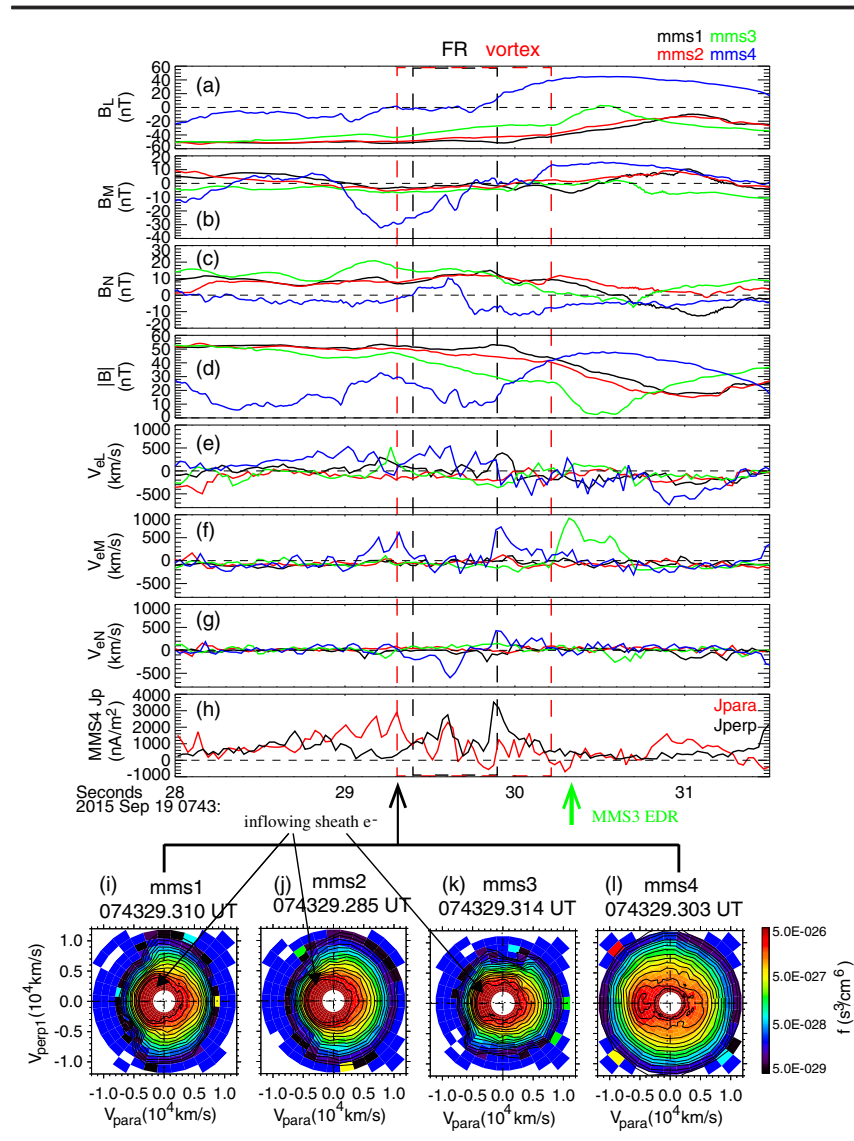

FIG. 2. Four spacecraft measurements in the vicinity of the secondary FR. From the top to bottom: magnetic field $B_{L}$ (a), $B_{M}$ (b), $B_{N}$ (c), and $|B|(\mathrm{d})$, electron bulk velocity $V_{e L}$ (e), $V_{e M}$ (f), and $V_{e N}(\mathrm{~g})$, parallel (red) and perpendicular (black) electric current observed by MMS4 (h). Panels (i)-(l) show the 2D cuts $V_{\text {para }}-V_{\text {perp1 }}$ of the $3 \mathrm{D}$ electron velocity distributions around 07:43:29.3 UT from the four spacecraft. $V_{\text {perp } 1}$ is defined as $(\mathbf{b} \times \mathbf{v}) \times \mathbf{b}(\mathbf{b}$ and $\mathbf{v}$ are unit vectors of the magnetic field and the electron bulk velocity, respectively).

If tearing instability was responsible for the generation of this FR and the associated flow reversal, MMS4 would have recorded a negative $V_{e L}$ corresponding to a positive $B_{N}$ and vice versa [20]. However, MMS4 observed a positive $V_{e L}$ corresponding to a positive $B_{N}$ and a negative $V_{e L}$ corresponding to a negative $B_{N}$, which is opposite to the $V_{e L}$ and $B_{N}$ variation expected in the tearing mode scenario. This indicates that the FR was not generated by the tearing instability, and hence the two opposite electron jets in $\mathbf{L}$ were not produced by two $X$ lines bounding the FR.

The nearly simultaneous electron flow reverses in the $V_{e L}$ and $V_{e N}$ components (marked by the red dashed box in Fig. 2) suggests that a flow vortex may have passed MMS4. If the flow reversals were caused by a flow vortex then the vortex must be clockwise because $V_{e L}$ changed from positive to negative when MMS4 crossed the vortex from the magnetosheath side to the magnetospheric side, i.e., along the $-\mathbf{N}$ direction. A clockwise in-plane electron current would cause an out-of-plane magnetic field enhancement in the $-\mathbf{M}$ direction, which is consistent with the MMS observation as shown in Fig. 2(b). In addition, the vortex must have moved in the $+\mathbf{L}$ direction in order to match the observed polarity change of $V_{e N}$. This is also consistent with the moving direction of the secondary FR inferred above, suggesting that the two structures were likely coupled with each other. Figure 3(d) shows a schematic view of the expected flow vectors along the spacecraft trajectory as it crosses an electron vortex in the $L-N$ plane. The observed variation of the flow vectors agrees well with the expected flow vectors variation as the crossing of a vortex. Hence, we conclude that MMS4 detected a clockwise electron flow vortex which was moving in the $+\mathbf{L}$ direction.

Figures 2(i)-2(1) show the 2D slices of the 3D electron velocity distributions at about 07:43:29.3 UT when the vortex was observed. The inflowing sheath population together with the sign of $B_{L}$ enables us to determine whether the spacecraft was in the $+\mathbf{L}$ or $-\mathbf{L}$ side of the $X$ line [18]. We found that MMS1\&2\&3 were on the $-\mathbf{L}$ side of the $X$ line because the inflowing sheath electrons have $V_{\text {para }}<0$ [Figs. 2(i)-2(k)] in the region where $\mathrm{B}_{L}<0$. MMS4, which was located between MMS2 and MMS3 in the $L$ direction, observed dramatically diminishing sheath electrons and increasing hot exhaust electrons [Fig. 2(1)]; hence, MMS4 stayed in the exhaust region $-\mathbf{L}$ side of the $X$ line. This also means that the vortex and secondary FR were on the $-\mathbf{L}$ side of the $X$ line.

There are significant electron flow shears in both the $L$ and $N$ directions, as $\Delta V_{e L}$ and $\Delta V_{e N}$ are nearly $1000 \mathrm{~km} / \mathrm{s}$, where $\Delta V_{e}$ is the flow shear speed. Theoretically, the flow shear speed must be sufficiently large to cause the electron $\mathrm{K}-\mathrm{H}$ instability unstable since the characteristic growth rate must exceed the whistler frequency in the electron current sheet [10]. The unstable criterion is $\Delta V_{e}>\left|C_{A e}\right| / 2$, where $C_{A e}$ is the shear component of the local electron Alfvén speed. Figures 3(b) and 3(c) show the comparison between $V_{e L}$ and $\left|C_{A e L}\right| / 2$, and $V_{e N}$ and $\left|C_{A e N}\right| / 2$, where $C_{A e L}$ and $C_{A e N}$ is the local electron Alfvén speed along the $\mathbf{L}$ and $\mathbf{N}$ directions, respectively. $\left|C_{A e L}\right| / 2$ was smaller than $\Delta V_{e L} \sim$ $1000 \mathrm{~km} / \mathrm{s}$ as $B_{L} \sim 0$ during 07:43:29.4-07:43:29.9 UT. Moreover, $\Delta V_{e N} \sim 1000 \mathrm{~km} / \mathrm{s}$ exceeds $\left|C_{A e N}\right| / 2$ in this time interval. Hence, the unstable criterion for the electron $\mathrm{K}-\mathrm{H}$ instability is satisfied within the vortex in both the $L$ and $N$ directions. We should note that the flow shear was neither parallel nor perpendicular to the background magnetic field; hence, this case is intermediate between the conditions of flow shear parallel to magnetic field and transverse to magnetic field.

The vortex was only observed by MMS4 implying that the spatial size of this vortex was less than the minimum distance between MMS4 and the other spacecraft, which was about $50 \mathrm{~km} \sim 1.1 d_{i}$ in the $\mathbf{N}$ direction. We estimated that the MP moved in the $\mathbf{N}$ direction with a speed of 


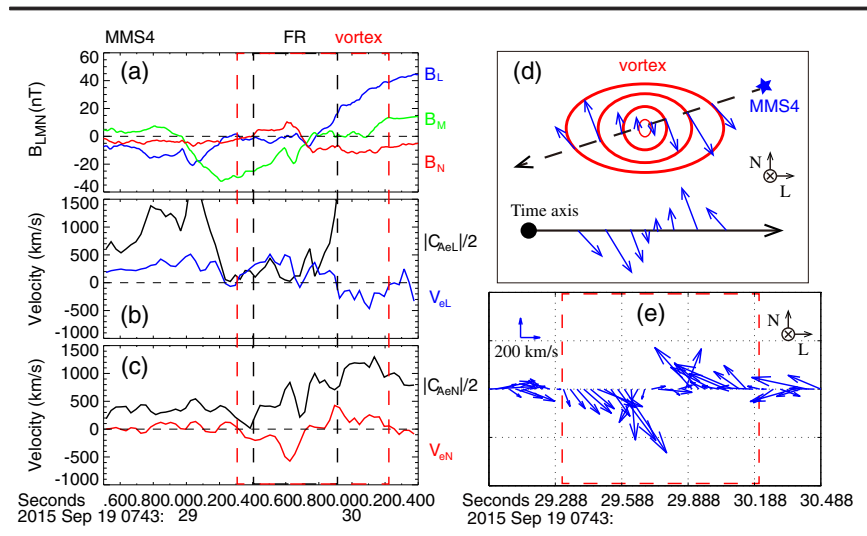

FIG. 3. Evidence of the electron K-H vortex. (a) Three components of magnetic field, (b)-(c) electron bulk velocity in the $L$ and $N$ direction, and the half local electron Alfvén speed along the $L$ and $N$ directions, (d) a schematic showing the trajectory of MMS4 across a vortex and the corresponding electron flow vectors in the $L-N$ plane, (e) electron flow vectors (by subtracting the mean velocity) in the $L-N$ plane as a function of time observed by MMS4.

$\sim 44 \mathrm{~km} / \mathrm{s}$ by using multiple-spacecraft timing analysis based on the magnetic field $B_{L}$ [21]. Assuming that the vortex moved with the same speed as the MP along $\mathbf{N}$, we found that the vortex was about $40 \mathrm{~km} \sim 0.9 d_{i}$ in $\mathbf{N}$. In addition, the average electron bulk velocity in the $\mathbf{L}$ direction within the vortex was about $70 \mathrm{~km} / \mathrm{s}$. It is positive since the electron jet speed toward the $X$ line is larger than the speed of the jet outward from the $X$ line. This net speed is the propagation speed of the vortex, based on which we deduced its size in $\mathbf{L}$ as $65 \mathrm{~km} \sim 1.4 d_{i}$. Since the FR was embedded within the vortex, it is reasonable to assume that the FR propagated with the same velocity as the vortex. Hence, the size of the FR was about $35 \mathrm{~km} \sim 0.8 d_{i}$ in $\mathbf{L}$ and $22 \mathrm{~km} \sim 0.5 d_{i}$ in $\mathbf{~}$.

As shown in Fig. 2(f), in the vicinity of the K-H vortex, there are two distinct enhancements of $V_{e M}$ observed by MMS4 at 07:43:29.3 and 07:43:29.9 UT, respectively. The second flow enhancement was primarily perpendicular to the magnetic field, while the first one was primarily antiparallel to the magnetic field. Figure 4 presents a close view of the second flow enhancement. It was located within the $\mathrm{K}-\mathrm{H}$ vortex and coincident with the trailing edge of the secondary FR. The electron perpendicular bulk speed $V_{e \perp}$ deviates from the $E \times B$ drift speed [Fig. 4(c)] at the peak of $V_{e M}$, which is a signature of the slippage of electrons with respect to the magnetic field [22].

Furthermore, the measure of electron nongyrotropy [Fig. 4(e)], which is a new scalar measure of the gyrotropy of a pressure tensor [23], also maximizes at the $V_{e M}$ enhancement with a peak value near 0.1. This means that electrons were effectively unmagnetized. Electron velocity distributions corresponding to the peak of $V_{e M}$ show crescentlike structures in the plane perpendicular to the magnetic field [Figs. 4(f) $-4(\mathrm{~g})]$. These distributions are

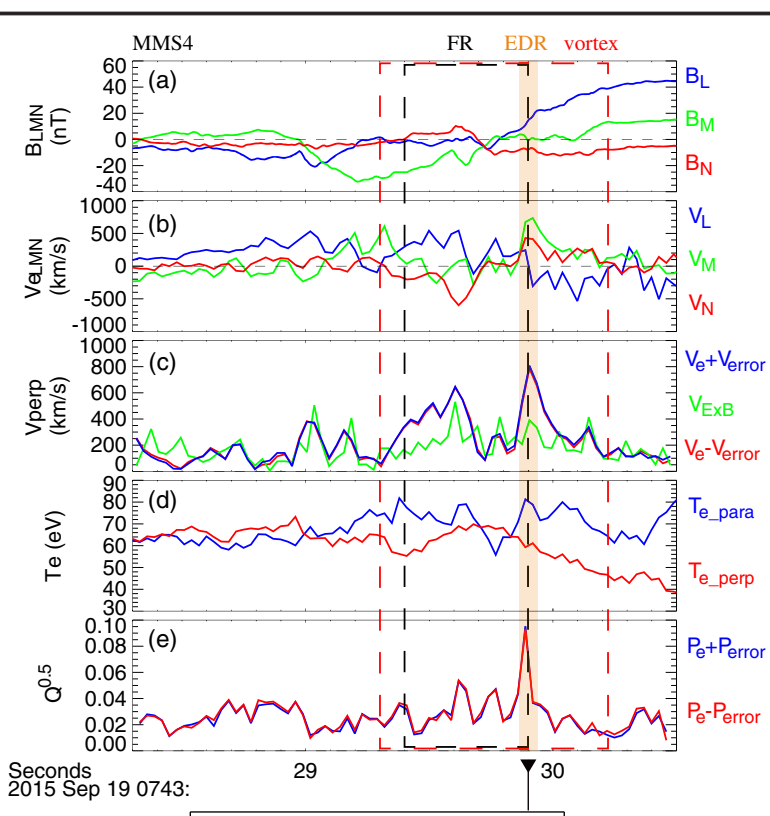

(f) 074329.903 UT

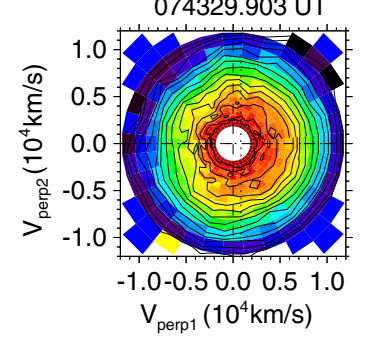

(g)

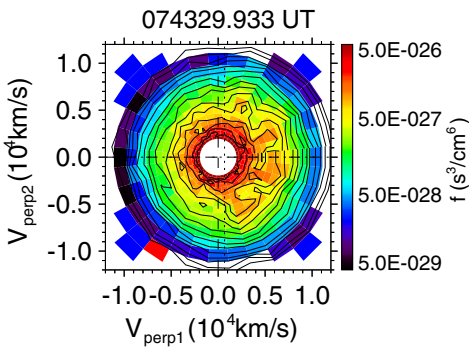

FIG. 4. Observation of an EDR (marked by the orange shadow) by MMS4. (a) Three components of magnetic field and (b) electron bulk flow, (c) electron perpendicular velocity and $V_{E \times B}$, (d) electron temperatures parallel and perpendicular to $B$, (e) electron nongyrotropy $Q^{1 / 2}$. Red and black traces in panel (c) and (e) indicate the values that were calculated by using the lower and upper limit of the measured quantities, respectively. Panels (f) and (g) show the $2 \mathrm{D}$ cuts $V_{\text {perp1 }}-V_{\text {perp2 }}$ of the $3 \mathrm{D}$ electron velocity distributions in the vicinity of the EDR. Here $V_{\text {perp1 }}$ is defined in Fig. 2 and $V_{\text {perp2 }}$ is defined as $\mathbf{b} \times \mathbf{v}$.

consistent with the characteristic electron velocity distribution of EDRs with small guide field as reported in Refs. [18,24-25]. This type of distribution emerges around electron-scale field reverse layers [26]. We should note that the fingerlike structures in the velocity distribution in Fig. 4(g) are due to the rapid variations of the magnetic field or velocity distributions within the $30 \mathrm{~ms}$ sample. The figurelike structures are superposition of four different velocity distributions sampled at $7.5 \mathrm{~ms}$ resolution [14]. The above evidences undoubtedly indicate that this $V_{e M}$ enhancement corresponds to an EDR.

This EDR was not the EDR associated with the primary $X$ line because this one was located on the $-\mathbf{L}$ side of the $X$ line as mentioned above. We believe that it was a secondary EDR induced by the electron K-H vortex. Although $\left|V_{e \perp}\right|$ and $\left|V_{E \times B}\right|$ were also different within the FR from 
07:43:29.3 to $07: 43: 29.6$ UT, the corresponding electron nongyrotropy is not obvious; hence, this region was unlikely an EDR.

Electron temperature was anisotropic inside the vortex as shown in Fig. 4(d). $T_{e \|}$ is greater than $T_{e \perp}$ in most part of the vortex, whereas $T_{e \perp}$ reaches a peak at the center of the secondary FR. This means that electrons were preferentially heated in the perpendicular direction within the FR. On the contrary, electrons were heated in the parallel direction within the secondary EDR, which is similar to the previous observations [25].

In summary, we present the first direct evidence that a secondary FR was generated by electron vortex as a result of the electron K-H instability during magnetic reconnection. This secondary FR was embedded within the electron vortex and adjacent to a secondary EDR. The polarity change of $B_{N}$ and associated electron flow variation exclude the tearing instability as a possible generation mechanism for this FR. The electron flows shear speed satisfied the unstable criterion of the electron $\mathrm{K}-\mathrm{H}$ instability [10]. These suggest that the formation of the secondary FR resulted from the evolution of the electron K-H vortex in the MP current sheet. The size of the electron vortex was $\sim 1.4 d_{i}$ in $\mathbf{L}$ and $\sim 0.9 d_{i}$ in $\mathbf{N}$, while the size of the secondary FR was $\sim 0.8 d_{i}$ in $\mathbf{L}$ and $\sim 0.5 d_{i}$ in $\mathbf{N}$.

We propose the following scenario for the generation of the subion scale FR in the IDR: (i) magnetic reconnection produces intense electron shear flow; (ii) the shear flow, which satisfies the unstable condition of the electron K-H instability, leads to the formation of electron vortex; (iii) the vortex induces a secondary reconnection in the vicinity of the primary $X$ line and finally leads to the formation of the secondary FR. This scenario is consistent with recent kinetic simulations [10,11].

Kelvin-Helmholtz instability is ubiquitous in planetary physics and astrophysics. It is an important mechanism for plasma transport across the MP [27]. Besides, KelvinHelmholtz instability can generate vortex flow, which significantly suppresses microturbulence and changes the transport mode of plasma and fluid physics [28]. Here we show that K-H instability is also fundamental in reconnection, though it operates on a much smaller scale. We suggest that, besides the tearing instability, secondary instabilities such as electron K-H instability are essential ingredients of reconnection. Since the K-H instability is triggered by an electron jet within the IDR, our results provide significant new insights on the electron physics of reconnection.

We thank the entire MMS team and MMS Science Data Center for providing the high quality data for this study. This work was supported by National Natural Science Foundation of China (NSFC) Grants No. 41674144, No. 41464007, No. 41331070,
No. 41522405, and No. 41774154 and the Science Foundation of Jiangxi Province (Grants No. 2014BAB202008 and No. 20142BCB23006).

* Corresponding author. tangrx@gmail.com Corresponding author. monmentum82@gmail.com Corresponding author. dengxhua@gmail.com

[1] J. F. Drake, M. Swisdak, H. Che, and M. A. Shay, Nature (London) 443, 553 (2006).

[2] H. Karimabadi, W. Daughton, and J. Scudder, Geophys. Res. Lett. 34, L13104 (2007).

[3] S. Y. Huang et al., Geophys. Res. Lett. 39, L11103 (2012).

[4] M. Zhou, Y. Pang, X. Deng, S. Huang, and X. Lai, J. Geophys. Res. 119, 6177 (2014).

[5] M. Zhou et al., Phys. Rev. Lett. 119, 055101 (2017).

[6] W. Daughton, V. Roytershteyn, H. Karimabadi, L. Yin, B. J. Albright, B. Bergen, and K. J. Bowers, Nat. Phys. 7, 539 (2011).

[7] M. Zhou, X. H. Deng, and S. Y. Huang, Phys. Plasmas 19, 042902 (2012).

[8] J. P. Eastwood et al., Geophys. Res. Lett. 43, 4716 (2016).

[9] R. Wang, Q. Lu, A. Du, and S. Wang, Phys. Rev. Lett. 104, 175003 (2010).

[10] R. L. Fermo, J. F. Drake, and M. Swisdak, Phys. Rev. Lett. 108, 255005 (2012).

[11] C. Huang, Q. Lu, F. Guo, M. Wu, A. Du, and S. Wang, Geophys. Res. Lett. 42, 7282 (2015).

[12] J. L. Burch, T. E. Moore, R. B. Torbert, and B. L. Giles, Space Sci. Rev. 199, 5 (2016).

[13] C. T. Russell et al., Space Sci. Rev. 199, 189 (2016).

[14] C. Pollock et al., Space Sci. Rev. 199, 331 (2016).

[15] P. A. Lindqvist et al., Space Sci. Rev. 199, 137 (2016).

[16] R. E. Ergun et al., Space Sci. Rev. 199, 167 (2016).

[17] B. U. O. Sonnerup and M. Scheible, No. SR-001, ISSI Scientific Reports (ESA Publications Division, Noordwijk, Netherlands 1998), Chap. 1, p. 185.

[18] L. J. Chen et al., Geophys. Res. Lett. 43, 6036 (2016).

[19] S. Wang et al., Geophys. Res. Lett. 43, 4850 (2016).

[20] J. P. Eastwood, D. G. Sibeck, J. A. Slavin, M. L. Goldstein, B. Lavraud, M. Sitnov, S. Imber, A. Balogh, E. A. Lucek, and I. Dandouras, Geophys. Res. Lett. 32, L11105 (2005).

[21] C. T. Russell, M. M. Mellott, E. J. Smith, and J. H. King, J. Geophys. Res. 88, 4739 (1983).

[22] M. V. Goldman, D. L. Newman, and G. Lapenta, Space Sci. Rev. 199, 651 (2016).

[23] M. Swisdak, Geophys. Res. Lett. 43, 43 (2016).

[24] M. Hesse, Y.-H. Liu, L.-J. Chen, N. Bessho, M. Kuznetsova, J. Birn, and J. L. Burch, Geophys. Res. Lett. 43, 2359 (2016).

[25] J. L. Burch et al., Science 352, aaf2939 (2016).

[26] G. Lapenta et al., J. Geophys. Res. 122, 2024 (2017).

[27] H. Hasegawa, M. Fujimoto, T.-D. Phan, H. Rème, A. Balogh, M. W. Dunlop, C. Hashimoto, and R. TanDokoro, Nature (London) 430, 755 (2004).

[28] Z. X. Wang, J. Q. Li, J. Q. Dong, and Y. Kishimoto, Phys. Rev. Lett. 103, 015004 (2009). 